\title{
Holomorphic diffeomorphisms of semisimple homogeneous spaces
}

\author{
Árpád Tóth and Dror Varolin
}

\begin{abstract}
We study the infinite-dimensional group of holomorphic diffeomorphisms of certain Stein homogeneous spaces. We show that holomorphic automorphisms can be approximated by generalized shears arising from unipotent subgroups. For the homogeneous spaces this implies the existence of Fatou-Bieberbach domains of the first and second kind, and the failure of the Abhyankar-Moh theorem for holomorphic embeddings.
\end{abstract}

\section{Introduction}

Complex analytic manifolds whose group of holomorphic diffeomorphisms acts transitively have been at the center of much research since Klein's Erlangen program. Almost as early on, studying these symmetries proved itself a useful tool in the function theory of several complex variables. When the homogeneous space can be represented as a bounded symmetric domain in $\mathbb{C}^{n}$, the group of holomorphic diffeomorphisms preserves a natural metric, and is therefore finite dimensional. Poincaré computed these groups in the case of the ball and the bidisk, and was led to the failure of the Riemann mapping theorem in higher dimensions.

In this paper we study the analytic geometry of Stein manifolds that support a transitive action, by holomorphic diffeomorphisms, of a complex semisimple Lie group. Unlike the aforementioned bounded symmetric domains, these manifolds have holomorphic diffeomorphism groups that are infinite dimensional, and thus a different approach is required for their study. This work is based on certain connections between representation theory and properties of the entire group of holomorphic diffeomorphisms that we establish through further development of our earlier work [TV00].

Past work in the study of affine homogeneous spaces has concentrated mainly on algebraic automorphisms, invariant theory, and applications to algebraic geometry. Even in the simplest case of $\mathbb{C}^{n}$, the group of holomorphic diffeomorphisms had not been well understood until the groundbreaking work of Andersén and Lempert [And90, AL92]. Recall that a shear is a transformation of $\mathbb{C}^{n}$ that, after an affine change of coordinates, has the form $(w, z) \mapsto(z, w+b(z))$, where $(z, w) \in \mathbb{C}^{n-1} \oplus$ $\mathbb{C}$ and $b \in \mathcal{O}\left(\mathbb{C}^{n-1}\right)$. A slight, but important, generalization of these maps, that we called overshears in previous work, is to allow maps of the form $(w, z) \mapsto\left(z, e^{a(z)} w+b(z)\right)$, where $(z, w) \in \mathbb{C}^{n-1} \oplus \mathbb{C}$ and $a, b \in \mathcal{O}\left(\mathbb{C}^{n-1}\right)$. The Andersén-Lempert theorem states that when $n \geqslant 2$ every holomorphic diffeomorphism of $\mathbb{C}^{n}$ can be approximated uniformly on compact sets by compositions of overshears.

In a manner similar to $\mathbb{C}^{n}$, semisimple homogeneous spaces such as the smooth affine quadrics $Q_{n}=S O(n+1, \mathbb{C}) / S O(n, \mathbb{C})=\left\{x_{0}^{2}+\cdots+x_{n}^{2}=1\right\} \subset \mathbb{C}^{n+1}$ are very symmetric and large in

Received 25 May 2005, accepted in final form 11 January 2006.

2000 Mathematics Subject Classification 32M05, 32M17, 32M25, $22 \mathrm{E} 46$.

Keywords: homogeneous complex manifolds, automorphism groups of affine manifolds, complex vector fields, semisimple Lie groups and their representations.

The first author was supported by OTKA grant no. T049693. The second author was partially supported by NSF grant DMS0400909.

This journal is (C) Foundation Compositio Mathematica 2006. 


\section{HOLOMORPHIC DIFFEOMORPHISMS OF HOMOGENEOUS SPACES}

the complex analytic sense, and so it is difficult to say things about the group of holomorphic diffeomorphisms.

The density property is a notion capturing the intuitive idea that the group of holomorphic diffeomorphisms is as large as possible. Let $X$ be a complex manifold, and $\mathcal{X}_{\mathcal{O}}(X)$ the Lie algebra of holomorphic vector fields on $X$. We say that $X$ has the density property if the Lie subalgebra generated by the complete vector fields on $X$ is dense in $\mathcal{X}_{\mathcal{O}}(X)$ :

$$
\overline{\left\langle\xi \in \mathcal{X}_{\mathcal{O}}(X) ; \xi \text { is complete }\right\rangle}=\mathcal{X}_{\mathcal{O}}(X) \text {. }
$$

(See $\S 2$ for the definition of complete vector fields.)

In fact, we establish the density property for a large collection of semisimple homogeneous spaces, i.e. complex homogeneous spaces that are obtained as quotients of complex semisimple Lie groups by closed complex subgroups. We will be only interested in the case where the quotient space is Stein, which, by a result of Matsushima, occurs if and only if the cosets are translates of a reductive complex subgroup. The main result of this paper is the following theorem.

THEOREM 1.1. If $G$ is a complex semisimple Lie group of adjoint type and $K$ a reductive subgroup, then the homogeneous space $X=G / K$ has the density property.

Recall that a semisimple Lie group is of adjoint type if its center is trivial or, equivalently, if the adjoint representation is faithful. We were not able to establish the density property for all semisimple homogeneous spaces; the proof of our main theorem uses the adjoint condition in a crucial way.

The density property is of fundamental significance, and governs the geometry of the underlying space in various respects, some of which were discussed in [Var00, Var01]. By results of [Var00], we have the following corollaries of Theorem 1.1.

Corollary 1.2. Let $X$ be as in Theorem 1.1, and let $n=\operatorname{dim}_{\mathbb{C}} X$.

(i) There is an open cover of $X$ by open subsets each of which is biholomorphic to $\mathbb{C}^{n}$, i.e. is a Fatou-Bieberbach domain.

(ii) The space $X$ is biholomorphic to one of its proper open subsets.

(iii) Let $Y$ be a complex manifold with $\operatorname{dim}(Y)<\operatorname{dim}(X)$, such that there exists a proper holomorphic embedding $j: Y \hookrightarrow X$. Then there exists another proper holomorphic embedding $j^{\prime}: Y \hookrightarrow X$ such that, for any $\varphi \in \operatorname{Diff}_{\mathcal{O}}(X), \varphi \circ j(Y) \neq j^{\prime}(Y)$.

Here and below, $\operatorname{Diff}_{\mathcal{O}}(X)$ refers to the group of holomorphic diffeomorphisms of the complex manifold $X$. We avoid the more standard notation $A u t(X)$, and use the words holomorphic diffeomorphisms in place of the more common automorphisms for fear that, when Lie groups are in the picture, use of the word automorphism may cause confusion.

Knowing the density property for a given space has applications not only to the geometry of that space, but also to some associated spaces on which the density property is not known to hold. There are many results of this kind, but for the purpose of illustration we restrict ourselves to the following theorem, which is our second main result of this paper.

Theorem 1.3. Let $X$ be a semisimple homogeneous space of dimension $n$ and $Y$ a complex manifold of dimension less than $n$ such that there exists a proper holomorphic embedding $j: Y \hookrightarrow X$. Then there exists another proper holomorphic embedding $j^{\prime}: Y \hookrightarrow X$ such that, for any $\varphi \in \operatorname{Diff} \mathcal{O}(X)$, $\varphi \circ j(Y) \neq j^{\prime}(Y)$.

It is not known if the spaces $X$ to which Theorem 1.3 applies have the density property. They are all finite covers of the spaces in Theorem 1.1, and the proof of Theorem 1.3 uses only this fact. 


\section{Á. TÓth AND D. VAROLIN}

It is an open problem whether finite covers of Stein manifolds with the density property have the density property, and vice versa. If this turns out to be true, it would greatly simplify the proof of the main result of [TV00]. In fact, the general relationship between the density property and covers (finite or not) is still not well understood, with the most famous open problem being the question of whether or not there is a Fatou-Bieberbach domain in $\mathbb{C}^{*} \times \mathbb{C}^{*}$.

One of the two central ingredients in the proof of Theorem 1.1 is the general notion of shears, introduced in [Var99]. This notion, whose key ideas are recalled in $\S 2$, turns the verification of the density property on homogeneous spaces into a problem in representation theory of semisimple Lie groups - the second central ingredient.

The density property was introduced so as to allow generalization to other complex manifolds of the previously mentioned results of Andersén and Lempert [And90, AL92]. In the process, this definition also simplified the proofs of those results, even in the original setting. The theorems of Andersén and Lempert began as answers to questions raised by Rosay and Rudin in their foundational paper [RR88]. It was also realized (in part in [AL92], and more fully in the joint work of Forstnerič and Rosay [FR93]) that the density property could be used in many interesting analytic geometric constructions in $\mathbb{C}^{n}$. In this regard, there are definitions of the density property for more general Lie algebras of vector fields (see [Var01]), taking into account whatever additional geometry one cares about. For more details on this side of the story, we recommend the survey papers [Fors96] and [Ros99].

As it turns out, the notion of the density property transcends generalization for its own sake, bringing to light new features of the complex manifolds possessing this property; witness Corollary 1.2 and further results in [Var00].

The density property on a Stein manifold $X$ is a meaningful statement about the size of the group $\operatorname{Diff}_{\mathcal{O}}(X)$. Indeed, one way of measuring the size of this group is to look at its formal tangent space: the closure of the Lie algebra generated by the complete vector fields on $X$. The density property is the statement that this formal tangent space is as large as possible.

Some additional remarks are of interest.

Remark 1. Previously, there was some hope that the 'largest' spaces were determined by their topology and the identical vanishing of the Kobayashi infinitesimal pseudometric. This was formulated precisely as the following question by Diederich and Sibony [DS79] for the case of $\mathbb{C}^{n}, n \geqslant 2$ :

If $X$ is a Stein manifold that is diffeomorphic to $\mathbb{R}^{2 n}$ and has identically vanishing Kobayashi infinitesimal pseudometric, is $X$ biholomorphic to $\mathbb{C}^{n}$ ?

A recent counterexample of Fornæss [Forn03] answered this question in the negative. In view of Corollary 1.2 part (i), we have the following conjecture.

Conjecture 1.4. If $X$ is a Stein manifold that is diffeomorphic to $\mathbb{C}^{n}$ and has the density property, then $X$ is biholomorphic to $\mathbb{C}^{n}$.

Remark 2. From the analytic geometry point of view, Stein manifolds with the density property seem to share many geometric properties of rational algebraic varieties, and thus it is natural to ask the following question.

Question 1.5. Does every Stein manifold with the density property have a Moishezon compactification? Is there a compactification that is bimeromorphic to $\mathbb{P}_{n}$ ?

By combining Corollary 1.2 part (i) with a famous theorem of Kodaira [Kod72], one has that a surface with the density property that also admits a compactification must be rational. This is generally false in higher dimensions. 


\section{HolomorPhiC DIFFEOMORPHISMS OF HOMOGENEOUS SPACES}

This paper is organized as follows. In $\S 2$ we recall various known results that are needed in the proofs of our main theorems. Some of these results are standard, and others were developed in [Var99] and [TV00]. In $\S 3$ we extend results in [TV00] to reduce the study of the density property on complex semisimple homogeneous spaces of adjoint type to a problem in representation theory. Although much of $\S 5$ is a collection of facts, it is technically the most demanding part of the paper, and so in $\S 4$ we show how, in some specific cases, the density property can be established without recourse to the technical arguments and full-blown root system machinery of $\S 5$. The homogeneous spaces we use as examples are ubiquitous, appearing naturally in many mathematical constructions. The conclusion of the proof of Theorem 1.1 occupies $\S 5$, using a case-by-case analysis of the representation theoretic problem established in $\S 3$. The proof of Theorem 1.3 occupies the sixth and final section.

\section{Background material}

In this section, we state various results that are needed later. This is also an opportunity to establish some notation. Since all of the material in this section is contained elsewhere, we omit almost all proofs.

\subsection{Holomorphic vector fields}

A holomorphic vector field $\xi$ on a complex manifold $X$ is a holomorphic section of $T_{X}^{1,0}$, the holomorphic part of the complexified tangent bundle. We let $\mathcal{X}_{\mathcal{O}}(X)$ denote the set of holomorphic vector fields on $X$. Since $T_{X}^{1,0}$ is naturally isomorphic to the real tangent bundle $T_{X}$, we can identify $\xi$ with a real vector field that we continue to denote by $\xi$. As such, there is a flow $\varphi_{\xi}$ associated to $\xi$, which is defined on an open subset $\mathcal{U}$ of $\mathbb{R} \times X$ containing $\{0\} \times X$ in the following way: For $(t, p) \in \mathcal{U}, \varphi_{\xi}^{\mathrm{t}}(p)=c(t)$, where $c:(-a(p), b(p)) \rightarrow X$ is the unique maximal solution of the initial value problem

$$
\frac{d c}{d t}=\xi \circ c, \quad c(0)=p
$$

It follows from general ordinary differential equation theory that the map $p \mapsto \varphi_{\xi}^{\mathrm{t}}(p)$ is holomorphic.

We say that $\xi$ is complete if $\mathcal{U}=\mathbb{R} \times X$, i.e. if for each $p \in X$ one can solve $(*)$ for all $t \in \mathbb{R}$. In this case $\left\{\varphi_{\xi}^{\mathrm{t}} \mid t \in \mathbb{R}\right\}$ is a one parameter group of holomorphic diffeomorphisms of $X$. We say that $\xi$ is $\mathbb{C}$-complete if both $\xi$ and $i \xi$ are complete. Define the $\mathbb{C}$-flow of $\xi$ to be

$$
g_{\xi}^{s+i t}:=\varphi_{\xi}^{s} \circ \varphi_{i \xi}^{\mathrm{t}}
$$

If $\xi$ is $\mathbb{C}$-complete, then $\left\{g_{\xi}^{\zeta} \mid \zeta \in \mathbb{C}\right\}$ defines a holomorphic $\mathbb{C}$-action. In this paper, all complete vector fields are $\mathbb{C}$-complete, so we shall often drop the prefix $\mathbb{C}$, and still refer to $g_{\xi}$ as the flow of $\xi$, even though it is defined for 'complex time'.

With the operation $[\xi, \eta]=\xi \eta-\eta \xi, \mathcal{X}_{\mathcal{O}}(X)$ forms a Lie algebra. We can generate a Lie subalgebra of $\mathcal{X}_{\mathcal{O}}(X)$ using complete vector fields on $X$. We shall call any vector field in the closure of this subalgebra completely generated. In general, this subalgebra will not consist of complete vector fields. However, completely generated vector fields have the extraordinary property that their flows can be approximated (in the locally uniform and hence $\mathcal{C}^{k}$ topology) by holomorphic diffeomorphisms of $X$ [Var01]. This result can be proved as a combination of an approximation method, often attributed to Euler, and the following formulas. Let $\xi$ and $\eta$ be vector fields with flows $\varphi_{\xi}$ and $\varphi_{\eta}$ respectively:

$$
\begin{gathered}
\left.\frac{d}{d t}\right|_{t=0} \varphi_{\xi}^{\mathrm{t}} \circ \varphi_{\eta}^{\mathrm{t}}=\xi+\eta, \\
\left.\frac{d}{d t}\right|_{t=0+} \varphi_{\eta}^{-\sqrt{t}} \circ \varphi_{\xi}^{-\sqrt{t}} \circ \varphi_{\eta}^{\sqrt{t}} \circ \varphi_{\xi}^{\sqrt{t}}=[\xi, \eta] .
\end{gathered}
$$




\section{Á. TÓth AND D. VAROLin}

The approximation method of Euler can be stated as follows.

Theorem 2.1. Let $X$ be a manifold, and let $\xi$ be a vector field with flow $\varphi_{\xi}$ on $X$. Suppose that $F_{t}: X \rightarrow X$ is a $\mathcal{C}^{1}$ family of self maps such that $F_{0}=\mathrm{id}_{X}$ and

$$
\left.\frac{d}{d t}\right|_{t=0} F_{t}=\xi
$$

Then, in the compact-open topology, one has

$$
\lim _{N \rightarrow \infty} \underbrace{F_{t / N} \circ \cdots \circ F_{t / N}}_{N \text { times }}=\varphi_{\xi}^{\mathrm{t}} .
$$

Putting all of this together, we have the following theorem.

Theorem 2.2. Let $\xi$ be a vector field that lies in the closure of the Lie algebra generated by the complete vector fields on $X$. Let $K \subset X$ be compact, and let $t$ be such that the flow of $\xi$ is defined up to time $t$ on $K$. Then $\varphi_{\xi}^{\mathrm{t}} \mid K$ can be approximated, uniformly on $K$, by holomorphic diffeomorphisms of $X$.

\subsection{General shears}

In [Var99] the following fundamental proposition was proved.

Proposition 2.3. Let $\xi \in \mathcal{X}_{\mathcal{O}}(X)$ be $\mathbb{C}$-complete, and let $f \in \mathcal{O}(X)$. Then $f \cdot \xi$ is $\mathbb{C}$-complete if and only if $\xi^{2} f=0$.

One is thus naturally led to study the function spaces

$$
I^{1}(\xi)=\{f \in \mathcal{O}(X) \mid \xi f=0\} \quad \text { and } \quad I^{2}(\xi)=\left\{f \in \mathcal{O}(X) \mid \xi^{2} f=0\right\}
$$

consisting of holomorphic first and second integrals respectively.

Definition 2.4. Let $\xi \in \mathcal{X}_{\mathcal{O}}(X)$ be $\mathbb{C}$-complete. We call $f \cdot \xi$ a $\xi$-shear (respectively $\xi$-overshear) if $f \in I^{1}(\xi)$ (respectively $f \in I^{2}(\xi)$ ).

We will often refer to $\xi$-shears simply as shears, and similarly with overshears.

Remark. Let $X=\mathbb{C}^{n}$, let $\xi$ be a constant vector field and let $f \in I^{j}(\xi)$ for $j=1,2$. In this case, it is the time- 1 map of $f \xi$, rather than $f \xi$ itself, that is called a shear and overshear in the literature. In a sense, our shears are infinitesimal shears.

For a given complete vector field, the existence of first integrals is a classical problem and a highly nontrivial matter. The orbits of the vector field must sit fairly nicely together, generally speaking. For the existence of second integrals that are not first integrals, almost all the orbits must be biholomorphic to $\mathbb{C}$, and the orbit space must be extremely regular [Var99]. Nevertheless, in the case of complex (semisimple) Lie groups, many left invariant vector fields have a lot of first and second integrals.

\subsection{Semisimple Lie algebras}

The following notation will be used throughout the paper. We denote by $\mathfrak{s l}(2, \mathbb{C})$ the threedimensional complex Lie algebra with basis $\{E, F, H\}$ satisfying the commutation relations

$$
[H, E]=2 E \quad[H, F]=-2 F \quad[E, F]=H .
$$

By a representation $\rho$ of a Lie algebra $\mathfrak{g}$, we mean a vector space $V$ and a linear map $\rho: \mathfrak{g} \rightarrow$ $\operatorname{End}(V)$ satisfying

$$
\rho([\xi, \eta])=\rho(\xi) \rho(\eta)-\rho(\eta) \rho(\xi) .
$$




\section{HOLOMORPHIC DIFFEOMORPHISMS OF HOMOGENEOUS SPACES}

We will refer to such $V$ as $\mathfrak{g}$-spaces, or $\mathfrak{g}$-modules. Let $a d: \mathfrak{g} \rightarrow \operatorname{End}(\mathfrak{g})$ be the adjoint representation, i.e. the representation of $\mathfrak{g}$ on itself given by $\operatorname{ad}(\xi) \eta:=[\xi, \eta]$ for all $\xi, \eta \in \mathfrak{g}$.

For a general semisimple Lie algebra $\mathfrak{g}$, we fix a Cartan subalgebra $\mathfrak{h}$, i.e. a commutative subalgebra all of whose elements can be simultaneously diagonalized in the adjoint representation, say, and whose dimension is maximal.

Given any representation $V$ of $\mathfrak{g}$, a weight of $V$ is a linear functional $\lambda: \mathfrak{h} \rightarrow \mathbb{C}$ such that the subspace

$$
V_{\lambda}:=\{v \in V ; H v=\lambda(H) v \text { for all } H \in \mathfrak{h}\}
$$

is nonzero.

The roots of $\mathfrak{h}$ are the weights $\alpha$ of the adjoint representation, and their union forms a root system $\Phi$. (See [Bou81] for more on root systems.) We choose an ordering of the roots as follows. Let $H \in \mathfrak{h}$ be such that no root vanishes on $H$. If a root $\alpha$ satisfies $\alpha(H)>0$, we say that $\alpha$ is positive, and write $\alpha \in \Phi^{+}$. Among the positive roots, there is a subset $\Delta$ of so called simple roots, with the property that any positive root can be written as a linear combination of the simple roots, with nonnegative integer coefficients.

The simple roots form a basis for $\mathfrak{h}^{*}$, the dual vector space of $\mathfrak{h}$. Moreover, given a root $\alpha,-\alpha$ is also a root, and there are vectors $E_{\alpha} \in \mathfrak{g}_{\alpha}, F_{\alpha} \in \mathfrak{g}_{-\alpha}$ and $H_{\alpha} \in \mathfrak{h}$ such that the following hold.

(i) The Lie algebra spanned by $E_{\alpha}, F_{\alpha}$ and $H_{\alpha}$ is isomorphic to $\mathfrak{s l}(2, \mathbb{C})$, i.e. $E_{\alpha}, F_{\alpha}$ and $H_{\alpha}$ satisfy the commutation relations (1). We define the Lie algebra isomorphism

$$
\phi_{\alpha}: \mathfrak{s l}(2, \mathbb{C}) \rightarrow \operatorname{span}\left\{E_{\alpha}, F_{\alpha}, H_{\alpha}\right\} \quad \text { by } \quad \phi_{\alpha}\left\{\begin{array}{l}
E \\
F \\
H
\end{array}\right\}=\left\{\begin{array}{l}
E_{\alpha} \\
F_{\alpha} \\
H_{\alpha}
\end{array}\right\} .
$$

(ii) For all linearly independent roots $\alpha, \beta$,

$$
\alpha\left(H_{\beta}\right)=\frac{2 B(\alpha, \beta)}{B(\beta, \beta)},
$$

where $B(\alpha, \beta)=\operatorname{tr}\left(a d H_{\alpha} a d H_{\beta}\right)$ is the form on the root system associated to $\mathfrak{h}$ induced by the Killing form of $\mathfrak{g}$.

The collection of $H_{\alpha}, \alpha \in \Phi$, is thus also a root system $\Phi^{*}$, called the dual of $\Phi$.

\subsection{Homogeneous spaces}

A homogeneous space $X$ is a manifold that has a transitive action by a Lie group $G$. In our main theorems, we assume that $G$ is semisimple and of adjoint type. Thus we are dealing with groups having trivial center. On occasion, when it is more convenient to do so, we might represent homogeneous spaces in the form $\tilde{G} / K$, where $\tilde{G}$ has finite center contained in $K$. It is known that every complex semisimple Lie group is algebraic, i.e. it is isomorphic to a closed subgroup of $S L_{N}(\mathbb{C})$ defined by the vanishing of certain polynomials. By Cartan's theorem A, the algebra $\mathbb{C}[G]$, of polynomial functions on $G$ (where $G$ is viewed as a closed submanifold of $\operatorname{End}\left(\mathbb{C}^{N}\right)$ ), is dense in $\mathcal{O}(G)$. If $K$ is a subgroup of $G$, the quotient manifold $G / K=\{g K: g \in G\}$ admits a transitive action by $G, h: g K \mapsto h g K$, and every manifold that admits a transitive action by $G$ arises in this way. By Matsushima's theorem [Mat60], such a quotient $G / K$ is Stein if and only if $K$ is reductive.

We now turn to the realization of $G / K$ in the case when $K$ is a reductive subgroup of $G$. This is the 'if' part of the theorem mentioned above, and is a classical theorem going back to Weyl and Hilbert. We use the word reductive in an analytic sense: $K$ is reductive if it contains a compact subgroup $K_{0}$ such that $\mathfrak{k}=\mathfrak{k}_{0}+i \mathfrak{k}_{0}$, where $\mathfrak{k}$ and $\mathfrak{k}_{0}$ are the Lie algebras of $K$ and $K_{0}$ respectively. With this definition, we shall need following result of Weyl. For the proof, see [Mum74]. 


\section{Á. TÓth AND D. VAROLIN}

Theorem 2.5 (Weyl). Let $G$ be a semisimple group, and $K$ a reductive subgroup. Then there is a representation of $G$ in a finite dimensional vector space $V$, and an element $v \in V$, such that the orbit $X=G \cdot v$ of $v$ by $G$ is closed, and biholomorphic to $G / K$.

The realization of $G / K$, via Theorem 2.5 , as an orbit in a finite dimensional representation is used in the proof of our theorems. To see how, let $\rho$ be a linear representation of $G$ in a vector space $V$. To every $\xi \in \mathfrak{g}$, we can associate a complete holomorphic vector field $\vec{\xi}$ on $V$ defined by

$$
\vec{\xi} f(p)=\left.\frac{d}{d t}\right|_{t=0} f\left(\rho\left(e^{(t \xi)}\right) p\right) \quad f \in \mathcal{O}(V), p \in V .
$$

Evidently $\vec{\xi}$ is $\mathbb{C}$-complete. If we now let $f$ be a (holomorphic) defining function for a $G$-invariant closed submanifold $X$ of $V$, in a neighborhood $U$ (in $V$ ) of a point $p \in X$, then

$$
(d f \vec{\xi})_{p}=\vec{\xi} f(p)=\left.\frac{d}{d t}\right|_{t=0} f\left(\rho\left(e^{(t \xi)}\right) p\right)=0
$$

since, for $t$ small enough, $\rho\left(e^{(t \xi)}\right) p \in U \cap X$. This proves the following proposition.

Proposition 2.6. For every $\xi \in \mathfrak{g}$, the vector field $\vec{\xi}$ is tangent to any smooth $G$-orbit in $V$.

Remark. Note that Weyl's construction provides and embedding of $G / K$ into a linear space on which $G$, an adjoint group, acts. It follows that the weights admitted by this representation are in the root lattice.

\section{Reduction to representation theory}

The first part of this section reviews some of our work in [TV00]. We present a number of technical refinements that will be used in the proof of Theorem 1.1.

\subsection{Polynomial vector fields}

We begin by introducing an algebraic version of Definition 2.4. To this end, let $V$ be a $\mathfrak{g}$-module via a representation $\rho: \mathfrak{g} \rightarrow \operatorname{End}(V)$. The vector space $V \otimes \mathfrak{g}$ has a natural $\mathfrak{g}$-module structure, given by

$$
(\rho \otimes a d)(\xi) v \otimes \eta=\rho(\xi) v \otimes \eta+v \otimes[\xi, \eta] .
$$

Definition 3.1. We call $f \otimes \xi \in V \otimes \mathfrak{g}$ a $\mathfrak{g}$-shear (respectively $\mathfrak{g}$-overshear) if $\rho(\xi) f=0$ (respectively $\left.\rho(\xi)^{2} f=0\right)$.

Our goal, then, is to study whether or not, for a given $\mathfrak{g}$-space $V$, overshears generate $V \otimes \mathfrak{g}$. The simplest case to consider is the case $\mathfrak{g}=\mathfrak{s l}(2, \mathbb{C})$, and we did so in [TV00]. We now recall several results from there, beginning with the following theorem.

Theorem 3.2. If $\mathfrak{g}=\mathfrak{s l}(2, \mathbb{C})$ and $V$ is either $\mathbb{C}^{2}$ or an irreducible module whose dimension is odd, then $V$ is generated by its $\mathfrak{s l}(2, \mathbb{C})$-overshears.

Now, given a $\mathfrak{g}$-space $V$, we can also form the $\mathfrak{g}$-space $\mathbb{C}[V] \otimes \mathfrak{g}$, where $\mathbb{C}[V]$ is the algebra of polynomial functions on $V$. Remarkably, using Theorem 3.2 one has the following result.

Theorem 3.3. Let $\mathfrak{g}$ be semisimple and let $V$ be a $\mathfrak{g}$-space. Assume that $V^{*} \otimes \mathfrak{g}$ is generated by its $\mathfrak{g}$-overshears. Then $\mathbb{C}[V] \otimes \mathfrak{g}$ is generated by its $\mathfrak{g}$-overshears.

In our applications elements of $\mathfrak{g}$ are linear vector fields on $V$, and so when $f \in \mathbb{C}[V]$ we write $f X$ for $f \otimes X$. Definition 3.1 was extracted from this setup. 


\section{HOLOMORPHIC DIFFEOMORPHISMS OF HOMOGENEOUS SPACES}

Proof. We choose a basis $x_{i}$ of $V^{*}$ consisting of weight vectors. This choice defines monomial basis (consisting of weight vectors) of the finite dimensional vector spaces of polynomials of a given degree. It suffices to check that, for every monomial $f \in \mathbb{C}[V]$ and $X \in \mathfrak{g}$ of the form $F_{\alpha}, E_{\alpha}$ or $H_{\alpha}, f X$ is completely generated. In this case, $X$ lies in some subalgebra of $\mathfrak{g}$ that is isomorphic to $\mathfrak{s l}(2, \mathbb{C})$ via $\phi_{\alpha}$.

Note that if, with respect to $H_{\alpha}$, each $x_{i}$ has even weight or $f$ has even weight, then there is nothing to prove; the result follows from Theorem 3.2. Thus we may assume without loss of generality that $x_{1}$ has odd weight $\lambda$ and that $f=x_{1} \cdot g$ where $g \in \mathbb{C}[V]$ has even weight $2 k$. Moreover, we note that it suffices to assume that $X=E_{\alpha}, H_{\alpha}$ or $F_{\alpha}$.

Case 1: $\left(X=H_{\alpha}\right)$. Then

$$
\left[g H_{\alpha}, x_{1} H_{\alpha}\right]=(\lambda-2 k) x_{1} g H_{\alpha}=(\lambda-2 k) f H_{\alpha} .
$$

Since $g H_{\alpha}$ has even weight, it is generated by shears. The result follows from the fact that $\lambda-2 k$ is odd and hence not zero.

Case 2: $\left(X=E_{\alpha}\right)$. Then

$$
\begin{aligned}
{\left[g H_{\alpha}, x_{1} E_{\alpha}\right] } & =g H_{\alpha}\left(x_{1}\right) E_{\alpha}+f\left[H_{\alpha}, E_{\alpha}\right]-x_{1} E_{\alpha}(g) H_{\alpha} \\
& =\left(\lambda_{1}+2\right) f E_{\alpha}-x_{1} E_{\alpha}(g) H_{\alpha} .
\end{aligned}
$$

As in Case $1, g H_{\alpha}$ is generated by shears. By Case $1, x_{1} E_{\alpha}(g) H_{\alpha}$ is completely generated. This case now follows from the fact that $\lambda+2$ is odd and hence not zero.

Case 3: $\left(X=F_{\alpha}\right)$. The case where $X=F_{\alpha}$ is handled in a fashion similar to Case 2.

Let $M$ be a Stein manifold, which we think of as already embedded in some $\mathbb{C}^{n}$. Suppose we are given a Lie algebra $\mathfrak{g}<\mathcal{X}_{\mathcal{O}}\left(\mathbb{C}^{n}\right)$ of holomorphic vector fields on $\mathbb{C}^{n}$ that are all tangent to $M$ and that span the tangent space to $M$ at every point. We denote by $\mathcal{O}_{\text {Aff }}(M)$ the restriction to $M$ of polynomials in $\mathbb{C}^{n}$, and write $\mathcal{X}_{\text {Aff }}(M):=\mathcal{O}_{\text {Aff }}(M) \cdot \mathfrak{g}$, i.e. $\mathcal{X}_{\text {Aff }}(M)$ consists of those vector fields that are linear combinations of the vector fields in $\mathfrak{g}$ with coefficients in $\mathcal{O}_{\text {Aff }}(M)$. The next result, established in [TV00], is a useful sufficiency criterion for the density property.

TheOREm 3.4. Suppose the Lie algebra $\mathfrak{g}$ has the following properties.

(i) The Lie algebra $\mathfrak{g}$ consists of linear vector fields (i.e. if $f$ is a linear function and $\xi \in \mathfrak{g}$, then $\xi f$ is linear).

(ii) The Lie algebra $\mathfrak{g}$ is semisimple and consists of complete vector fields.

(iii) We have $\mathcal{O}(M) \cdot \mathfrak{g}=\mathcal{X}_{\mathcal{O}}(M)$.

(iv) If $f$ is a linear function and $\xi \in \mathfrak{g}$, then $f \xi$ is in the Lie algebra generated by complete vector fields.

Then $\mathcal{X}_{\text {Aff }}(M)$ is generated by complete vector fields. Thus, in particular, $M$ has the density property.

Proof. Cartan's theorem A implies that $\mathcal{X}_{\mathrm{Aff}}(M)$ is a dense subset of $\mathcal{X}_{\mathcal{O}}(M)$ in the locally uniform topology. By Theorem 3.3, $\mathbb{C}[V] \otimes \mathfrak{g}$ is generated by $\mathfrak{g}$-overshears, and this remains true in $\mathcal{X}_{\text {Aff }}(M)$ because restriction to $M$ preserves Lie brackets. Since the elements of $\mathfrak{g}$ are complete, so are their overshears, by Proposition 2.3.

Remark. We do not ask for $M$ to be parallelized by a basis of $\mathfrak{g}$; it may happen that $\operatorname{dim} \mathfrak{g}>\operatorname{dim}_{\mathbb{C}} M$.

As an immediate corollary of Theorem 3.4, we have the following result. 


\section{Á. TÓth AND D. VAROLIN}

Theorem 3.5. Let $G \rightarrow \operatorname{End}(V)$ be a representation such that $V^{*} \otimes \mathfrak{g}$ is generated by its $\mathfrak{g}$-overshears. Then any closed orbit of $G$ has the density property.

We make use of this theorem by realizing our manifold as a closed orbit of $G$ via Weyl's theorem 2.5. (Recall that a closed submanifold of a Stein manifold is Stein.)

\subsection{Quadratic vector fields}

In [TV00] Theorem 3.2 was sufficient for establishing criterion (iv) of Theorem 3.4, and thus the density property on complex semisimple Lie groups. We now proceed to analyze the situation in greater depth. Recall that criterion (iv) requires vector fields of the form

$$
f \xi, \quad f \in V^{*}, \xi \in \mathfrak{g}
$$

to be completely generated. After choosing a basis of $V$ consisting of weight vectors, and the base $\left\{E_{\alpha}, F_{\alpha}: \alpha \in \Phi^{+}\right\} \cup\left\{H_{\alpha}: \alpha \in \Delta\right\}$ of $\mathfrak{g}$, we are led to checking finitely many vector fields. However, deciding whether any of these vector fields is completely generated could be fairly difficult. In this section we investigate to what extent we can reduce the number of vector fields to be checked so as to obtain the desired result.

To present our sharpening of Theorem 3.4 we introduce the following definition.

Definition 3.6. Let $\mathfrak{g}$ be a semisimple Lie algebra. A weight is called extremal if its orbit under the Weyl group contains a multiple of a fundamental weight. Given a representation $V$ of $\mathfrak{g}$ a weight vector $v \in V$ is called extremal if the corresponding weight is extremal.

The term extremal comes from the fact that the dominant extremal weights lie in the extremal lines of the dominant Weyl chamber (the cone of linear functionals that are positive with respect to the ordering).

Let $M$ and $\mathfrak{g}$ be as in Theorem 3.4, and fix a Cartan subalgebra $\mathfrak{h}$ of $\mathfrak{g}$.

Theorem 3.7. Suppose the Lie algebra $\mathfrak{g}$ has the following properties.

(i) The Lie algebra $\mathfrak{g}$ is semisimple and consists of complete vector fields that are linear (in the sense of part (i) in Theorem 3.4).

(ii) We have $\mathcal{O}(M) \cdot \mathfrak{g}=\mathcal{X}_{\mathcal{O}}(M)$.

(iii) If $f$ is a linear function that is an extremal weight vector, with extremal weight $\lambda$, and $H_{i} \in \mathfrak{h}$ is a simple co-root such that $\lambda\left(H_{i}\right) \neq 0$, then $f H_{i}$ is in the Lie algebra generated by complete vector fields.

Then $\mathcal{X}_{\mathrm{Aff}}(M)$ is generated by complete vector fields. Thus, in particular, $M$ has the density property.

Remark. The reduction to $\mathfrak{h}$ is natural from the geometric point of view: the problematic vector fields are those whose orbit is $\mathbb{C}^{*}$. The extremality condition exploits the extra structure of semi-simple Lie algebras, and does not seem to have a geometric interpretation.

The proof of Theorem 3.7 is a series of somewhat technical observations. We will state these results for general $\mathfrak{g}$-modules $W$, since they do not rely on any specific properties of the $\mathfrak{g}$-space at hand, but the reader should keep in mind that in our application $W$ is the dual of this $\mathfrak{g}$-space. We begin with the following lemma.

Lemma 3.8. Let $\mathfrak{g}$ be a semisimple Lie algebra, $\mathfrak{h}<\mathfrak{g}$ a Cartan subalgebra, and $\rho: \mathfrak{g} \rightarrow \operatorname{End}(W)$ a representation of $\mathfrak{g}$ in $W$. Then

$$
W \otimes \mathfrak{g}
$$

is generated by $W \otimes \mathfrak{h}$ as $\mathfrak{g}$-module. 


\section{HOLOMORPHIC DIFFEOMORPHISMS OF HOMOGENEOUS SPACES}

Proof. Let $W_{0}$ be the submodule of $W \otimes \mathfrak{g}$ generated by $W \otimes \mathfrak{h}$. Given a root $\alpha$ and the corresponding co-root $H_{\alpha}$, choose a nonzero $X_{\alpha} \in \mathfrak{g}$ so that $\left[H_{\alpha}, X_{\alpha}\right]=2 X_{\alpha}$. The set of all such $X_{\alpha}$, together with $\mathfrak{h}$, span $\mathfrak{g}$. If now $w \in W$, then

$$
2 w \otimes X_{\alpha}=\left(X_{\alpha} w\right) \otimes H_{\alpha}-X_{\alpha}\left(w \otimes H_{\alpha}\right) \in W_{0},
$$

as desired.

The next lemma shows that the weights for only a few of the $\mathfrak{s l}(2, \mathbb{C})$-subalgebras $\left\langle X_{\alpha}, H_{\alpha}\right\rangle$ need to be even.

Lemma 3.9. Suppose $\rho: \mathfrak{g} \rightarrow \operatorname{End}(W)$ is a representation of a semisimple Lie algebra $\mathfrak{g}$, and $\mathfrak{h}$ is a Cartan subalgebra of $\mathfrak{g}$. Let $\lambda$ be a weight of $\mathfrak{h}$ in $W$ and $W_{\lambda}=\{w \in W ; H w=\lambda(H) w\}$ the corresponding subspace of $W$. If there exists a co-root $H_{\alpha}$ so that $\lambda\left(H_{\alpha}\right)$ is even and nonzero, then $W_{\lambda} \otimes \mathfrak{h}$ is contained in the submodule generated by the $\mathfrak{g}$-overshears.

Proof. Let $\alpha$ be such that $\lambda\left(H_{\alpha}\right)$ is positive and even. Since $\lambda\left(H_{\alpha}\right) \neq 0$, we have that

$$
\mathfrak{h}=\mathbb{C} H_{\alpha} \oplus \operatorname{ker} \lambda .
$$

Let $W^{\prime}$ be the smallest subspace of $W$ that is invariant under $\phi_{\alpha}(\mathfrak{s l}(2, \mathbb{C}))$ and contains $W_{\lambda}$. Under the action of $\phi_{\alpha}(\mathfrak{s l}(2, \mathbb{C})), W^{\prime}$ has only even weights, and thus by Theorem 3.2 it is generated by the $\phi_{\alpha}(\mathfrak{s l}(2, \mathbb{C}))$ overshears. These are automatically $\mathfrak{g}$-overshears, showing that

$$
W_{\lambda} \otimes \mathbb{C} H_{\alpha}
$$

is generated by $\mathfrak{g}$-overshears. On the other hand, the elements of

$$
W_{\lambda} \otimes \operatorname{ker} \lambda
$$

are overshears for the trivial reason that, for $H \in \operatorname{ker} \lambda$ and $w \in W_{\lambda}, H w=0$.

Lemma 3.10. Let $\lambda$ be a weight that is not a multiple of a fundamental weight. Then there exists a co-root $H_{\alpha}$ such that $\lambda\left(H_{\alpha}\right)$ is positive and even.

Proof. Without loss of generality we may assume that $\lambda$ is dominant. We will use the notation of the Planches (Tables) of [Bou81]. We let $\Phi$ denote the root system of $\mathfrak{g}$. For a root $\alpha$, let $H_{\alpha}$ be the corresponding co-root in $\mathfrak{h}$. The set $\left\{H_{\alpha} ; \alpha \in \Phi\right\}$ is a root system dual to $\Phi$. For a simple root $\alpha_{i}$, we denote $H_{\alpha_{i}}$ by $H_{i}$. With $\lambda$ as in the hypothesis, one may choose simple roots $\alpha_{i}$, $\alpha_{j}$, so that $\lambda\left(H_{i}\right)>0, \lambda\left(H_{j}\right)>0$. Choosing $i, j$ minimal with respect to this property, we have that $\lambda\left(H_{k}\right)=0$, for all $i<k<j$. Assume that $\lambda\left(H_{i}\right)$ and $\lambda\left(H_{j}\right)$ are odd. No matter which root system is being considered, $H_{i j}=\sum_{i \leqslant k \leqslant j} H_{k}$ is a co-root (although it might not be the co-root corresponding to $\left.\sum_{i \leqslant k \leqslant j} \alpha_{k}\right)$. Then

$$
\lambda\left(H_{i j}\right)=\lambda\left(H_{i}\right)+\lambda\left(H_{j}\right)
$$

so that $\lambda\left(H_{i j}\right)$ must be positive and even.

Proof of Theorem 3.7. By Lemma 3.8 it is enough to show that $f H$ is completely generated for $f \in V^{*}, H \in \mathfrak{h}$. We may assume without any loss of generality that $f$ is a simultaneous eigenvector of $\mathfrak{h}$ with weight $\lambda$, and that $H=H_{\alpha}$, for some $\alpha \in \Delta$. If $\lambda$ is extremal then $f H$ is either complete (if $\lambda(H)=0$ ) or else, by assumptions (i) and (ii) in Theorem 3.7, it is completely generated. We may therefore assume that $\lambda$ is not a multiple of a fundamental weight. Lemma 3.10 shows that there is a co-root $H^{\prime} \in \mathfrak{h}$ so that $\lambda\left(H^{\prime}\right)$ is even. By Lemma 3.9 the vector fields in $V_{\lambda}^{*} \otimes \mathfrak{h}$ are completely generated in view of Proposition 2.3. Therefore condition (iii) of Theorem 3.7 implies condition (iv) of Theorem 3.4, and the result follows. 


\section{Á. TÓth AND D. VAROLIN}

\section{Examples}

In this section we provide simplified proofs of the density property for some special examples. Interestingly, some of these examples lie beyond the scope of Theorem 1.1.

\subsection{Small representations}

Definition 4.1. A representation $V$ of $\mathfrak{s l}(2, \mathbb{C})$ is said to be bounded by 2 if every weight $n$ satisfies $|n| \leqslant 2$. More generally, a representation $V$ of a complex semisimple Lie group $G$ is bounded by 2 if, for any root $\alpha$, the restriction of $V$ to $\phi_{\alpha}(\mathfrak{s l}(2, \mathbb{C}))$ is bounded by 2 .

Remark. (i) The notion of boundedness by 2 is independent of the choice of Cartan subalgebra. (ii) Since the weights of $V^{*}$ are the negatives of the weights of $V, V^{*}$ is also bounded by 2 .

The next proposition shows that one can use representations bounded by 2 to produce examples of affine homogeneous spaces with the density property. In [TV00] we established that, if $V$ is a representation bounded by 2 , then $V \otimes \mathfrak{g}$ is generated by its $\mathfrak{g}$-overshears. In view of Theorem 3.4, we therefore have the following proposition.

Proposition 4.2. If $G$ is a semisimple Lie group admitting a representation $V$ that is bounded by 2 , then every closed $G$-orbit in $V$ has the density property.

Proposition 4.2 can be used to establish the density property in many cases. We now proceed to do this in several examples.

\subsection{Affine quadrics}

Consider the smooth affine subvariety of $\mathbb{C}^{n+1}$ given by

$$
Q_{n}=\left\{x_{0}^{2}+\cdots+x_{n}^{2}=1\right\} .
$$

Theorem 4.3. For any $n \geqslant 2$, the affine quadric $Q_{n}$ has the density property.

This follows from Proposition 4.2 and the fact that $Q_{n}$ is a closed orbit of the standard representation

$$
S O(n+1, \mathbb{C}) \rightarrow \operatorname{End}\left(\mathbb{C}^{n+1}\right),
$$

which is bounded by 1 . Note that this homogeneous space is not of adjoint type.

We will describe this example in some detail. The $n(n+1) / 2$ vector fields $X_{i j}, 0 \leqslant i<j \leqslant n$, given by

$$
X_{i j}:=x_{i} \partial_{j}-x_{j} \partial_{i}, \quad 0 \leqslant i<j \leqslant n,
$$

are tangent to $Q_{n}$ and generate the module $\mathcal{X}_{\text {Aff }}\left(Q_{n}\right)$. The relation

$$
\left[X_{i j}, X_{k l}\right]=\delta_{j k} X_{i l}-\delta_{i k} X_{j l}+\delta_{i l} X_{j k}-\delta_{j l} X_{i k}
$$

where $\delta_{i j}$ is the Kronecker delta, shows that the Lie algebra

$$
\mathfrak{g}=\operatorname{span}\left\{X_{i j} ; 0 \leqslant i<j \leqslant n\right\}
$$

is isomorphic to $\mathfrak{s o}(n+1, \mathbb{C})$. Let $k$ be the largest integer such that $2 k<n$. A Cartan subalgebra of $\mathfrak{g}$ is

$$
\operatorname{span}\left\{\sqrt{-1} X_{0,1}, \ldots, \sqrt{-1} X_{2 k, 2 k+1}\right\}
$$

and the weight vectors with nonzero weights are

$$
x_{0} \pm \sqrt{-1} x_{1}, \ldots, x_{2 k} \pm \sqrt{-1} x_{2 k+1},
$$

showing that this representation is bounded by 1 . 


\section{HOLOMORPHIC DIFFEOMORPHISMS OF HOMOGENEOUS SPACES}

\subsection{The space of Lagrangian splittings}

Let $V=\mathbb{C}^{2 n}$ with the standard symplectic form

$$
\langle x, y\rangle:=x^{\mathrm{t}} J y
$$

where $x^{\mathrm{t}}$ is the transpose of $x$ and

$$
J=\left[\begin{array}{cc}
0 & -I \\
I & 0
\end{array}\right]
$$

with the entries 0 and $I$ signifying the $n \times n$ zero and identity matrices. The symplectic group

$$
S p(2 n, \mathbb{C}):=\left\{g \in G L(2 n, \mathbb{C}): g^{\mathrm{t}} J g=J\right\}
$$

acts transitively on the Lagrangian subspaces of $V$, i.e. the subspaces of $V$ that are isotropic for $\langle$, and have maximal dimension. The set of all Lagrangian subspaces is a projective manifold, called the Lagrangian Grassmannian, and thus the stabilizer of any single Lagrangian is parabolic. Choose, for example, $V_{1}$ to be the span of the first $n$ standard basis vectors $e_{1}=[1,0,0, \ldots, 0,0]^{\mathrm{t}}, e_{2}=$ $[0,1,0, \ldots, 0,0]^{\mathrm{t}}, \ldots, e_{n}=[0,0,0, \ldots, 0,1]^{\mathrm{t}}$. The stabilizer of $V_{1}$ is the subgroup

$$
P=\left\{\left[\begin{array}{ll}
a & b \\
0 & d
\end{array}\right] \in S p(2 n, \mathbb{C})\right\} .
$$

Consider the set $\mathcal{L}(V)$ of Lagrangian decompositions of $V$, i.e. ordered pairs of Lagrangian subspaces $\left(L_{1}, L_{2}\right)$, so that $V=L_{1} \oplus L_{2}$. This set can also be given a holomorphic and even algebraic structure, and the resulting manifold $M$ is Stein, supporting a transitive action by $S p(2 n, \mathbb{C})$. If $V_{2}$ is the subspace generated by $e_{n+1}, \ldots, e_{2 n}$, then the stabilizer

$$
L=\left\{\left[\begin{array}{ll}
a & 0 \\
0 & d
\end{array}\right] \in S p(2 n, \mathbb{C})\right\}=\left\{\left[\begin{array}{cc}
a & 0 \\
0 & \left(a^{\mathrm{t}}\right)^{-1}
\end{array}\right] ; a \in G L(n, \mathbb{C})\right\}
$$

of the pair $\left(V_{1}, V_{2}\right)$ is a Levi component of $P$. Indeed, $L$ is reductive,

$$
U=\left\{\left[\begin{array}{ll}
I & b \\
0 & I
\end{array}\right] \in S p(2 n, \mathbb{C})\right\}
$$

is unipotent, and $P=L U$.

Now $L$ is the centralizer of

$$
\lambda=\left[\begin{array}{cc}
I & 0 \\
0 & -I
\end{array}\right] \in M_{2 n}(\mathbb{C}),
$$

and so $M$ can be identified with the orbit of $\lambda$ in $M_{2 n}(\mathbb{C})$, the space of $2 n \times 2 n$ matrices, on which $S p(2 n, \mathbb{C})$ acts by conjugation. One checks that $\lambda \in \mathfrak{s} \mathfrak{p}(2 n, \mathbb{C})$, the Lie algebra of $S p(2 n, \mathbb{C})$, and so the orbit of $\lambda$ lies entirely in $\mathfrak{s p}(2 n, \mathbb{C})$. The orbit is closed and is described as

$$
\left\{\left[\begin{array}{cc}
x & y \\
z & -x^{\mathrm{t}}
\end{array}\right]: y^{\mathrm{t}}=y, z^{\mathrm{t}}=z, x^{2}+y z=I\right\} \text {. }
$$

Since the adjoint representation of $\mathfrak{s p}(2 n, \mathbb{C})$ is bounded by 2, Proposition 4.2 implies the following theorem.

Theorem 4.4. For every symplectic vector space $V$, the manifold $\mathcal{L}(V)$ of all Lagrangian splittings of $V$ has the density property.

The space of Lagrangian splittings is an example of a symplectic homogeneous space, which we now discuss more generally. 


\section{Á. TÓth AND D. VAROLIN}

\subsection{Symplectic homogeneous spaces}

For more details on this brief overview, we refer the reader to [Bou81]. Let $G$ be a semisimple Lie group. A closed subgroup $P$ is called parabolic if $G / P$ is projective. We give a very brief description of $P$ in terms of the root system of the Lie algebra of $G$.

Let $\Phi^{+}$denote the set of positive roots, and $\Delta$ the set of simple roots. Let $\mathfrak{n}$ be the subalgebra generated by $X_{\alpha}, \alpha \in \Phi^{+}$, and $\mathfrak{b}$ the subalgebra generated by $\mathfrak{n}$ and $\mathfrak{h}$.

Let $B$ denote the connected closed subgroup of $G$ associated to the Lie algebra $\mathfrak{b}$. The quotient $G / B$ is a projective manifold, and, by a famous theorem of Borel, $B$ is a minimal parabolic subgroup in the sense that every parabolic subgroup is conjugate to one that contains $B$. Therefore, up to inner automorphisms, it is enough to describe those subgroups $P$ whose Lie algebra $\mathfrak{p}$ contains $\mathfrak{b}$. This is done as follows. Since $\mathfrak{p}$ contains $\mathfrak{h}$, there is basis of $\mathfrak{p}$ consisting of eigenvectors of $\mathfrak{h}$, and the set of negative roots admitted by $\mathfrak{p}$, i.e. the set

$$
\left\{\alpha \in \Phi ; \exists X_{\alpha} \in \mathfrak{p} \text { such that } \forall H \in \mathfrak{h},\left[H, X_{\alpha}\right]=\alpha(H) X_{\alpha} \text { and }-\alpha \in \Phi^{+}\right\},
$$

must be closed under addition. Let $H$ be an element of $\mathfrak{h}$ such that $\alpha(H) \geqslant 0$ for all $\alpha \in \Phi^{+}$, and let $\Phi^{\prime}=\{\alpha \in \Phi: \alpha(H)=0\}$. Then the subspace with basis

$$
\left\{X_{\alpha}: \alpha \in \Phi^{\prime}\right\} \cup\left\{H_{\alpha}: \alpha \in \Delta\right\} \cup\left\{X_{\alpha}: \alpha \in \Phi^{+} \backslash \Phi^{\prime}\right\}
$$

is a subalgebra, and so it is the Lie algebra of a parabolic subgroup. This is the only way parabolic subalgebras can arise. Moreover, when $H \neq 0$, the algebra constructed here is proper. In that case $P=L U$, where the Lie algebra of $L$ is generated by

$$
\left\{X_{\alpha}: \alpha \in \Phi^{\prime}\right\} \cup\left\{H_{\alpha}: \alpha \in \Delta\right\},
$$

and thus $L$ is reductive. The Lie algebra of $U$ is generated by $\left\{X_{\alpha}: \alpha \in \Phi^{+} \backslash \Phi^{\prime}\right\}$, showing that $U$ is unipotent. Therefore the Levi component $L$ is the centralizer of the semisimple element $H$ used in the construction of $\mathfrak{p}$.

For the rest of this section we view $G$ as a subgroup of $\operatorname{End}(\mathfrak{g})$ through the adjoint representation. Let $P=L U$ be a parabolic subgroup, where $L$ arises as the stabilizer of a semisimple element $H \in \mathfrak{h}$. Since, at the Lie algebra level, our representation is the derivative of the group action at the identity, the Lie subgroup of $G$ with Lie algebra equal to the centralizer of $H$ is the stabilizer of $H$ in the adjoint action. Therefore $M:=G / L$ is realized as the orbit of $H$ in $\mathfrak{g}$ under $A d$, and it is well known that the adjoint orbit of a semisimple element is an algebraic submanifold of $\mathfrak{g}$ (see [Bou81]).

Theorem 4.5. The closed adjoint orbit $M=G / L \subset \mathfrak{g}$ described above has the density property.

To prove Theorem 4.5 it is enough to show that the conditions of Theorem 3.4 are satisfied. Note that if $\mathfrak{g}$ has no factors of type $G_{2}$, the adjoint representation is bounded by 2 and we could apply Proposition 4.2 to these cases. However, the following result covers all the cases and employs a uniform argument that avoids using the classification of complex semisimple Lie algebras. The techniques are modifications of those used in the proof of Theorem 6.3 in [TV00].

THEOREM 4.6. The adjoint representation is $\mathfrak{g}$-completely generated.

Proof. Let $G$ be the adjoint group with Lie algebra $\mathfrak{g}$. In view of Theorem 3.3, it suffices to show that, for each $x \in \mathfrak{g}$ and $\varphi \in \mathfrak{g}^{*}, \varphi \otimes x$ is generated by overshears.

Recall that for a representation $V$, the action of $\mathfrak{g}$ on the dual $V^{*}$ is given by $x \varphi(v)=-\varphi(x v)$.

Because $\mathfrak{g}$ is semisimple, it admits a nondegenerate Killing form, and thus an isomorphism between the adjoint representation and its dual. Using the notation of $\S 2$, this isomorphism is given explicitly as follows. Let

$$
e_{\alpha}(x)=B\left(E_{\alpha}, x\right) \quad \text { for all } x \in \mathfrak{g},
$$

and define $f_{\alpha}$ and $g_{\alpha}$ similarly. 


\section{HOLOMORPHIC DIFFEOMORPHISMS OF HOMOGENEOUS SPACES}

Without loss of generality, we may assume that $\varphi$ is any of the $e_{\alpha}, h_{\alpha}, f_{\alpha}$ where $\alpha \in \Phi^{+}$. Moreover, because of the symmetry between $e_{\alpha}$ and $f_{\alpha}$, it suffices to prove only that $h_{\alpha} \otimes E_{\beta}, h_{\alpha} \otimes$ $H_{\beta}, f_{\alpha} \otimes E_{\beta}, e_{\alpha} \otimes H_{\beta}$ and $e_{\alpha} \otimes E_{\beta}$ are generated by overshears. In what follows, $n$ will be used to denote some integer that may vary from case to case.

Case 1: $h_{\alpha} \otimes H_{\beta}$ is a shear and $h_{\alpha} \otimes E_{\beta}$ is an overshear. For the first,

$$
H_{\beta} h_{\alpha}(x)=-h_{\beta}\left(\left[H_{\alpha}, x\right]\right)=-B\left(H_{\beta},\left[H_{\alpha}, x\right]\right)=B\left(\left[H_{\alpha}, H_{\beta}\right], x\right)=0,
$$

and for the second,

$$
E_{\beta}^{2} h_{\alpha}(x)=-B\left(H_{\alpha},\left[E_{\beta},\left[E_{\beta}, x\right]\right]\right)=-B\left(\left[\left[H_{\alpha}, E_{\beta}\right], E_{\beta}\right], x\right)=0 .
$$

Case 2: $f_{\alpha} \otimes E_{\beta}=\frac{1}{2}\left(F_{\alpha}\left(h_{\alpha} \otimes E_{\beta}\right)+n h_{\alpha} \otimes H_{\beta}\right)$, so in view of case $1, f_{\alpha} \otimes E_{\beta}$ is generated by overshears.

Case 3: $e_{\alpha} \otimes H_{\beta}=-\frac{1}{2}\left(E_{\alpha}\left(h_{\alpha} \otimes H_{\beta}\right)+n h_{\alpha} \otimes E_{\beta}\right)$, so in view of case $1, e_{\alpha} \otimes H_{\beta}$ is generated by overshears.

Case 4: $e_{\alpha} \otimes E_{\beta}=-\frac{1}{2}\left(E_{\beta}\left(e_{\alpha} \otimes H_{\beta}\right)+c e_{\alpha+\beta} \otimes H_{\beta}\right)$ for some constant $c$, so in view of cases 1 and $3, e_{\alpha} \otimes E_{\beta}$ is generated by overshears.

This completes the proof.

It is known [GS77] that, if $X$ is a semisimple homogeneous space that admits a symplectic form, then there is a semisimple homogeneous space of the type $G / L$ described above, and a finite holomorphic covering map $\pi: X \rightarrow G / L$. Thus one can apply Theorem 1.3 to obtain the following corollary, which we believe is worth stating separately.

Corollary 4.7. Let $X$ be a semisimple homogeneous space that admits a symplectic form and $Y$ a complex manifold with $\operatorname{dim}(Y)<\operatorname{dim}(X)$ such that there exists a proper holomorphic embedding $j: Y \hookrightarrow X$. Then there exists another proper holomorphic embedding $j^{\prime}: Y \hookrightarrow X$ such that, for any $\varphi \in \operatorname{Diff}_{\mathcal{O}}(X), \varphi \circ j(Y) \neq j^{\prime}(Y)$.

\section{Proof of Theorem 1.1}

In this section we establish Theorem 1.1 using Theorem 3.7. In order to reduce the former to the latter, we provide proofs for a number of simple but perhaps more esoteric facts about root systems.

We start with some obvious reductions, whose proofs are left to the reader.

LEMMA 5.1.

(a) If $V$ and $W$ are $\mathfrak{g}$-spaces such that $V \otimes \mathfrak{g}$ and $W \otimes \mathfrak{g}$ are generated by their $\mathfrak{g}$-overshears, then $(V \oplus W) \otimes \mathfrak{g}$ is generated by its $\mathfrak{g}$-overshears.

(b) Suppose Lie algebras $\mathfrak{g}_{1}$ and $\mathfrak{g}_{2}$, and $\mathfrak{g}_{i}$-spaces $V_{i}, i=1,2$, are given, so that $V_{i} \otimes \mathfrak{g}_{i}$ are generated by their $\mathfrak{g}_{i}$-overshears. Then $\left(V_{1} \otimes V_{2}\right) \otimes\left(\mathfrak{g}_{1} \oplus \mathfrak{g}_{2}\right)$ is generated by its $\mathfrak{g}_{1} \oplus \mathfrak{g}_{2}$-overshears.

The representation $\rho$ of $\mathfrak{g}_{1} \oplus \mathfrak{g}_{2}$ on $V_{1} \otimes V_{2}$ is given by

$$
\rho\left(\xi_{1} \oplus \xi_{2}\right)\left(v_{1} \otimes v_{2}\right)=\rho_{1}\left(\xi_{1}\right) v_{1} \otimes v_{2}+v_{1} \otimes \rho_{2}\left(\xi_{2}\right) v_{2} .
$$

Using Lemma 5.1, it suffices to consider the case where $\mathfrak{g}$ is simple and $V$ is an irreducible $\mathfrak{g}$-module. To this end, the main result of this section is the following theorem.

TheOREM 5.2. Let $\mathfrak{g}$ be a simple Lie algebra, and let $V$ be an irreducible $\mathfrak{g}$-space whose highest weight is in the root lattice of $\mathfrak{g}$. Then $V \otimes \mathfrak{g}$ is generated by its $\mathfrak{g}$-overshears. 


\section{Á. TÓth AND D. VAROLin}

In view of Theorem 3.7 the main task is to handle the case of multiples of the fundamental weights. Such weights are sparse in the root lattice, but they do arise. In the case of representations with highest weight in the root lattice, the situation is dealt with by making use of the following facts. (Again, we use the notation of the Planches (Tables) of [Bou81].)

Lemma 5.3. Let $\Phi$ be a simple root system, $B=\left\{\alpha_{1}, \ldots, \alpha_{l}\right\}$ a set of simple roots in $\Phi$, and $\left\{\omega_{1}, \ldots, \omega_{l}\right\}$ the corresponding set of fundamental weights. Let $m \neq 0$ be such that $\lambda=m \omega_{i}$ is in the root lattice.

(a) Assume that $\Phi$ is of type $A_{2 l+1}, B_{l}, C_{l}, D_{l}, E_{7}, E_{8}, F_{4}$ or $G_{2}$ and that $\omega_{i}$ is arbitrary. Then there exists some root $\alpha$, so that $\lambda\left(H_{\alpha}\right)$ is a nonzero even integer.

(b) Assume that $\Phi=E_{6}$, and that $\lambda=m \omega_{i}$ for $i=2,3,4,5,6$. Then there exists some root $\alpha$, so that $\lambda\left(H_{\alpha}\right)$ is a nonzero even integer.

(c) If $\Phi=A_{2 l}$ and $\lambda=m \omega_{i}$ for some $i$, or if $\Phi=E_{6}$ and $\lambda=m \omega_{1}$, then $m \omega_{i} \pm \alpha_{i}$ is not an integral multiple of a fundamental weight.

Proof. (a) and (b). Recall that, for any $\lambda \in \mathfrak{h}^{*}$,

$$
\lambda\left(H_{\alpha}\right)=\frac{2(\lambda, \alpha)}{(\alpha, \alpha)}
$$

where $(\cdot, \cdot)$ is some multiple of the Killing form. To simplify the computations, when working with the Planches (Tables) of [Bou81], we will use the ordinary scalar product in $\mathbb{R}^{n}$, which in all cases is a scalar multiple of the Killing form.

(i) When $\Phi=A_{2 l+1}$, and $\lambda=m \omega_{i}$ is in the root lattice, then $(2 l+2) \mid m$, and so $m$ is even.

(ii) When $\Phi$ is of type $B_{l}$, then $\omega_{1}\left(H_{e_{1}}\right)=2$ and, for all $i>1, \omega_{i}\left(H_{e_{1}+e_{2}}\right)=2$.

(iii) When $\Phi$ is of type $C_{l}$, then, for all $i>1, \omega_{i}\left(H_{e_{1}+e_{2}}\right)=2$. When $i=1, \omega_{1} \notin \Phi$, but $2 \omega_{1} \in R$, forcing $m$ to be even.

(iv) When $\Phi$ is of type $D_{l}$ and $1<i<l-1, e_{1}+e_{2}$ still works for $\omega_{i}$. None of $\omega_{1}, \omega_{l-1}, \omega_{l}$ is in $\Phi$, and their index in the root lattice is either 2 or 4 , forcing $m$ to be even if $m \omega_{1}, m \omega_{l-1}$ or $m \omega_{l}$ is to be in the root lattice.

(v) When $\Phi$ is of type $E_{6}, E_{7}, E_{8}$ or $G_{2}$ we use the fact that

$$
\check{\Phi}=\left\{\frac{2 \alpha}{(\alpha, \alpha)}: \alpha \in \Phi\right\}=\Phi .
$$

First assume that the pair $\left(\Phi, \omega_{i}\right)$ is different from the pairs $\left(E_{6}, \omega_{6}\right)$ and $\left(E_{7}, \omega_{7}\right)$. Then one finds roots $\alpha=\sum_{j} c_{j} \alpha_{j}$ so that $c_{i}$ is even. For $\left(E_{6}, \omega_{6}\right)$, note that $m$ must be divisible by 3 , and that $\frac{1}{2}\left(\sum_{j=1}^{5} e_{j}-e_{6}-e_{7}+e_{8}\right)$ works. When $\Phi=E_{7}$, once again $m \omega_{7}$ will be in the root lattice only for even $m$.

(vi) Finally, when $\Phi$ is of type $F_{4}, e_{1}$ works when $i=1,2$ and 4 , and $\frac{1}{2}\left(e_{1}+e_{2}+e_{3}-e_{4}\right)$ works when $i=3$.

(c) This follows from the Euclidean geometry of the root systems $A_{2 \ell}$ and $E_{6}$. One could also consult the relevant Planches (Tables) in [Bou81].

The last detail we need is the following generalization of Theorem 3.2.

Proposition 5.4. Let $V$ be an irreducible representation of $\mathfrak{s l}(2, \mathbb{C})$, and suppose $v \in V$ is such that $H v=\lambda v$ for some $\lambda \neq 0$. If neither $E$ nor $F$ annihilates $v$, then $V \otimes \mathfrak{s l}(2, \mathbb{C})$ is generated by $v \otimes H$ and the $\mathfrak{s l}(2, \mathbb{C})$-overshears of $V \otimes \mathfrak{s l}(2, \mathbb{C})$. 


\section{HOLOMORPHIC DIFFEOMORPHISMS OF HOMOGENEOUS SPACES}

Proof. If $j$ is the largest integer so that $E^{j} v \neq 0$, then $E^{j} v \otimes E$ and $E^{j-1} v \otimes E$ are overshears, and so $F^{j+1}\left(E^{j} v \otimes E\right)$ and $F^{j}\left(E^{j-1} v \otimes E\right)$ are in $V$. Since $F E^{j} v=(\lambda+2 j) E^{j-1} v$, one can easily prove, using induction, that

$$
F^{j+1}\left(E^{j} v \otimes E\right)=\left(F^{j+1} E^{j} v\right) \otimes E-(j+1)\left(F^{j} E^{j} v\right) \otimes H+j(j+1) F^{j-1} E^{j} v \otimes F
$$

and

$$
F^{j}\left(E^{j-1} v \otimes E\right)=\left(F^{j} E^{j-1} v\right) \otimes E-j\left(F^{j-1} E^{j-1} v\right) \otimes H-j(j-1) F^{j-2} E^{j-1} v \otimes F .
$$

These identities make sense, as $j \geqslant 2$. It follows that the three vectors

$$
v \otimes H, \quad F^{j+1}\left(E^{j} v \otimes E\right) \quad \text { and } \quad F^{j}\left(E^{j-1} v \otimes E\right)
$$

are linearly independent. Since $V \otimes \mathfrak{s l}(2, \mathbb{C})$ is generated by its three-dimensional $\lambda$ eigenspace, the proof is complete.

Proof of Theorem 5.2. Let $W$ be the submodule of $V \otimes \mathfrak{g}$ generated by $\mathfrak{g}$-overshears. By Lemma 3.8 we need to show that, when $H \in \mathfrak{h}$ and $v$ is a weight vector of weight $\lambda, v \otimes H \in W$. By Lemmas 3.9 and 3.10 we know that this is true if $\lambda$ is not an integral multiple of a fundamental weight. The same arguments show that $v \otimes H \in W$ even when $\lambda=m \omega_{i}$ when $m=0$, or when $\omega_{i}$ is one of the fundamental weights listed in Lemma 5.3(a).

In the remaining cases, we can assume that $H$ is one of the co-roots corresponding to a simple root; these were denoted $H_{i}$ above. Consider the subalgebra generated by the elements $E_{i}, F_{i}, H_{i}$, which we denote $\phi_{i}(\mathfrak{s l}(2, \mathbb{C}))$. Let $M$ be the smallest subspace of $V$ that contains $V_{\lambda}$ and is invariant under $\phi_{i}(\mathfrak{s l}(2, \mathbb{C}))$, and write $M=\bigoplus_{\nu} M_{\nu}$, where each $M_{\nu}$ is irreducible as a $\phi_{i}(\mathfrak{s l}(2, \mathbb{C}))$-module. By complete reducibility, $V_{\lambda}=\bigoplus_{\nu} M_{\nu} \cap V_{\lambda}$. Choose $v_{\nu} \in V_{\lambda} \cap M_{\nu}$.

If both $E_{i}^{2} v_{\nu}$ and $F_{i}^{2} v_{\nu}$ are zero, but $E_{i} v_{\nu}$ and $F_{i} v_{\nu}$ are nonzero, then the representation $M_{\nu}$ is isomorphic to the adjoint representation of $\mathfrak{s l}(2, \mathbb{C})$, and so $H_{i} v_{\nu}=0$, i.e. $m=0$. When $E_{i} v_{\nu}=0$, $v_{\nu} \otimes H_{i}=F_{i}\left(E_{i} v_{\nu} \otimes E_{i}\right)-v_{\nu} \otimes E_{i} \in W$, and a similar computation works if $F_{i} v_{\nu}=0$. Therefore, we may assume that either $E_{i}^{2} v_{\nu} \neq 0$ or $F_{i}^{2} v_{\nu} \neq 0$. Moreover, by symmetry, it suffices to assume that $E_{i}^{2} v_{\nu} \neq 0$. In this case, since $\lambda+\alpha_{i}$ is not a multiple of a fundamental weight (Lemma 5.3), we can apply Proposition 5.4 with $v=E_{i} v_{\nu}$, and conclude from Lemmas 3.9 and 3.10 that $V_{\lambda+\alpha_{i}} \otimes H \subset W$, as desired.

Taking into account the remark at the end of $\S 2$ and the remark following Theorem 3.3, Theorem 1.1 now follows from Theorems 3.5, 2.5 and 5.2.

\section{Lifting arguments - proof of Theorem 1.3}

In the proof of Theorem 1.3 we make use of two ideas, due respectively to Winkelmann [Win01] and to Forstnerič, Globevnik and Rosay [FGR96]. We shall describe the needed versions of these ideas below, but we will be brief with certain parts of the proof, as the proofs that appear elsewhere can be modified to produce the facts that we need here.

We say that two discrete sets $S$ and $T$ on a complex manifold $M$ are equivalent if there exists $f \in \operatorname{Diff}_{\mathcal{O}}(M)$ such that $f(S)=T$. If no such diffeomorphism exists, we say that $S$ and $T$ are inequivalent. A theorem of Winkelmann [Win01] asserts the existence of inequivalent sets on any Stein manifold. All discrete sets we deal with are assumed to be infinite.

We begin with the following proposition, which can be established easily from Winkelmann's theorem and a counting argument.

Proposition 6.1. Suppose that $\pi: \tilde{X} \rightarrow X$ is a covering map between Stein manifolds. Then there exist discrete sets $S, T \subset \tilde{X}$ that are not equivalent, such that $\pi \mid S$ and $\pi \mid T$ are one-to-one. 


\section{Á. TÓth AND D. VAROLIN}

Following the method of [FGR96], one needs next to prove the following result.

Theorem 6.2. Let $\pi: \tilde{X} \rightarrow X$ be a finite normal covering map of Stein manifolds with deck group $\Gamma$, and suppose $X$ has the density property. Suppose $K \subset \tilde{X}$ is compact, holomorphically convex, and satisfies $\gamma K=K$ for all $\gamma \in \Gamma$. Let $A \subset K$ be a finite set, $p, q \in \tilde{X}-K$ and $\epsilon>0$. Then there exists $F \in \operatorname{Diff}_{\mathcal{O}}(\tilde{X})$ such that

(i) $F(p)=q$,

(ii) $F \mid A=$ id, and

(iii) $\sup _{x \in K} \operatorname{dist}(F(x), x)<\epsilon$.

The proof of Theorem 6.2 is based on Theorem 2.2. We start with the construction of a vector field on a cover from a vector field on the base, in such a way that completeness is inherited. In fact, this is the situation with the usual lifting of maps in covering space theory: $d \pi: T_{\tilde{X}} \rightarrow T_{X}$ is also a covering space, and the section $\xi: X \rightarrow T_{X}$ lifts to a map $\xi^{\prime}: X \rightarrow T_{\tilde{X}}$ because there are no topological obstructions. The map $\xi^{\prime}$ then factors through $\pi$, and the factor $\tilde{\xi}: X \rightarrow T_{X}$ is actually a section. Rather than proving all of this topologically, we shall construct the vector field $\tilde{\xi}$ directly in the case where the cover is normal. This is the only case needed in our setting.

To this end, let $p \in \tilde{X}$. Suppose that $\pi^{-1} \pi p=\left\{p, q_{2}, \ldots, q_{d}\right\}$, and let $U_{1}, \ldots, U_{d}$ be neighborhoods of $p, q_{2}, \ldots, q_{d}$, respectively, that are mutually disjoint and such that $\pi \mid U_{j}: U_{j} \rightarrow U$ is a diffeomorphism. (In particular, $\pi\left(U_{i}\right)=\pi\left(U_{j}\right)$.) We set

$$
\tilde{\xi}_{p}:=d\left(\pi \mid U_{1}\right)^{-1} \xi_{\pi p}
$$

We leave it to the reader to check that this vector field is well defined. Let $\Gamma$ be the deck group of the covering $\pi$. Since for any $\gamma \in \Gamma$ one has $\pi \gamma=\pi$, the vector field $\tilde{\xi}$ so defined is invariant, and thus is the lift mentioned above.

Lemma 6.3. Let $\xi$ be a holomorphic vector field on $X$. If $\xi$ is sufficiently close to zero on a compact set $K \subset X$, then $\tilde{\xi}$ is small on the compact set $\pi^{-1} K$. Moreover, if $\xi$ is complete, then the vector field $\tilde{\xi}$ is complete on $\tilde{X}$.

Proof. The size assertion is obvious. To see the completeness assertion, note that, in fact, one can lift every integral curve of $\xi$ to $M$. It is then an easy calculation to see that the lifted curve is an integral curve of $\tilde{\xi}$. Thus the latter is complete.

Proof of Theorem 6.2. Consider the set $\pi K \subset X$ and the two points $\pi p, \pi q \in X$. First observe that $\pi K$ is holomorphically convex. Indeed, if this is not so, then there is a function $f \in \mathcal{O}(X)$ such that, for some $x \notin \pi K,|f(x)|<\sup _{\pi K}|f|$, and then $f \circ \pi$ would show that $K$ is not holomorphically convex, which is a contradiction.

Let $c:[0,1+\delta] \rightarrow X$ be a smooth curve such that

(a) $c(0)=\pi p$ and $c(1)=\pi q$,

(b) the lift of $c$ to $\tilde{X}$ starting at $p$ reaches $q$ at time 1 , and

(c) $c([0,1+\delta]) \cap \pi K=\emptyset$.

We define $F_{t}: K \times\{c(t)\} \rightarrow X$ by

$$
F_{t}(x)= \begin{cases}x, & x \in K, \\ c(t), & x=p .\end{cases}
$$

Consider the time-dependent vector field

$$
\eta_{t}=\frac{d F_{t}}{d t} \circ F_{t}^{-1}
$$




\section{HolomorPhiC DIFFEOMORPHISMS OF HOMOGENEOUS SPACES}

Let $\eta(x, t)=\eta_{t}(x)$. Since $\pi K$ is holomorphically convex, a theorem of Stolzenberg implies that $\pi K \times[0,1+\delta]$ is holomorphically convex. It follows that we can approximate $\eta(x, t)$ by some $\xi \in \mathcal{O}\left(X \times \mathbb{C}, T_{X}\right)$ uniformly on $\pi K \times[0,1+\delta]$. Let $\xi_{t}(x)=\xi(x, t)$. Since $X$ has the density property, we may assume that $\xi_{t}$ is completely generated. Also, by approximation, the integral curve of $\xi_{t}$ starting at $\pi p$ at $t=0$ is arbitrarily close to $\pi q$ at time $t=1$.

We now lift $\xi_{t}$ to a time-dependent, $\Gamma$-invariant vector field $\tilde{\xi}_{t}$ on the cover $\tilde{X}$. By Lemma 6.3 and the functoriality of $\mathcal{X}_{\mathcal{O}}$ with respect to mappings we see that $\xi_{t}$ is completely generated. An application of Theorem 2.2 gives a holomorphic diffeomorphism $\Phi$ that carries $p$ arbitrarily close to $q$. By using a lifting of an implicit function-type argument as in [Var00, Theorem 3.1], we may correct $\Phi$ so that it carries $p$ exactly to $q$. This completes the proof.

Theorem 1.3 is an immediate consequence of Proposition 6.1 and the following theorem.

Theorem 6.4. Let $f: Y \hookrightarrow \tilde{X}$ be a proper holomorphic embedding of Stein manifolds with $\operatorname{dim}(Y)<\operatorname{dim}(\tilde{X})$, and let $S \subset \tilde{X}$ be any discrete subset such that $\pi \mid S$ is $1-1$. Then there exists an embedding $f^{\prime}: Y \hookrightarrow \tilde{X}$ such that $f^{\prime}(Y) \supset S$.

Proof. Let

$$
L_{0}=\emptyset \subset L_{1} \subset \operatorname{interior}\left(L_{2}\right) \subset L_{2} \subset \operatorname{interior}\left(L_{3}\right) \subset L_{3} \subset \cdots \subset \tilde{X}
$$

be a nested family of compact, holomorphically convex sets such that

$$
\tilde{X}=\bigcup_{\ell \geqslant 1} L_{\ell} \quad \text { and } \quad \gamma L_{\ell}=L_{\ell} \text { for all } \gamma \in \Gamma .
$$

(Such an exhaustion is easy to construct. Indeed, one takes an exhaustion by $\Gamma$-invariant compact sets, and then replaces these invariant compact sets by their holomorphic hulls. It is elementary to see that the hull of an invariant compact set is also invariant.)

We decompose $S$ into disjoint finite sets $S_{1}, S_{2}, \ldots$ defined by the requirement that

$$
S_{j} \subset L_{j}-L_{j-1} \text {. }
$$

Let $f_{0}:=f$, and suppose we have obtained an embedding $f_{j-1}: Y \rightarrow \tilde{X}$ such that

$$
f_{j-1}(\Sigma) \supset S_{1} \cup \cdots \cup S_{j-1} .
$$

We now apply Theorem 6.2 repeatedly to get $f_{j}$. To this end, enumerate

$$
S_{j}=\left\{s_{1, j}, \ldots, s_{N_{j}, j}\right\} .
$$

Let $K_{1, j}=L_{j}$, let $A_{1, j}=S_{1} \cup \cdots \cup S_{j-1}$, and let $p \in f_{j-1} \sigma-K_{1, j}$ and $q=s_{1, j}$. An application of Theorem 6.2 gives us $F_{j, 1} \in \operatorname{Diff}_{\mathcal{O}}(\tilde{X})$ with the properties stated there. Next, let $2 \leqslant \ell \leqslant N_{j}$ and suppose we have obtained $F_{j, \ell-1}$. Let $K_{\ell, j}=K_{\ell-1, j} \cup s_{\ell-1, j}$ and $A_{\ell, j}=A_{\ell-1, j} \cup s_{\ell-1, j}$, let

$$
p \in F_{\ell-1, j} \circ \cdots \circ F_{1, j} \circ f_{j-1}(\Sigma)-K_{\ell, j},
$$

and let $q=s_{\ell, j}$. Then, again, Theorem 6.2 gives us $F_{\ell, j} \in \operatorname{Diff}_{\mathcal{O}}(\tilde{X})$ with the properties stated there.

We now define

$$
f_{j}:=F_{N_{j}, j} \circ \cdots \circ F_{1, j} \circ f_{j-1} .
$$

It takes some additional care, in the construction of the $F_{k, j}$, to guarantee that $f_{j} \rightarrow f^{\prime}$ and that $f^{\prime}$ is proper. The details can be carried out in exactly the same way as in [FGR96]. 


\section{HOLOMORPHIC DIFFEOMORPHISMS OF HOMOGENEOUS SPACES}

\section{REFERENCES}

And90 E. Andersén, Volume preserving automorphisms of $\mathbb{C}^{n}$, Complex Variables 14 (1990), 223-235.

AL92 E. Andersén and L. Lempert, On the group of holomorphic automorphisms of $\mathbb{C}^{n}$, Invent. Math. 110 (1992), 371-388.

Bou81 N. Bourbaki, Éléments de mathématique. Groupes et algèbres de Lie (Masson, Paris, 1981), Chapters 4-6.

DS79 K. Diederich and N. Sibony, Strange complex structures on Euclidean space, J. reine angew. Math. 311/312 (1979), 397-407.

Forn03 J. E. Fornæss, Short $\mathbb{C}^{k}$, in Complex analysis in several variables - Memorial conference of Kiyoshi Oka's Centennial Birthday, Adv. Stud. Pure Math., vol. 42 (Math. Soc. Japan, Tokyo, 2004), 95-108.

Fors96 F. Forstnerič, Holomorphic automorphisms of $\mathbb{C}^{n}:$ a survey, in Proc. Complex Analysis and Geometry, eds V. Ancona, E. Ballico and A. Silva, Lecture Notes in Pure and Applied Mathematics, vol. 173 (Marcel Dekker, New York, 1996), 173-200.

FGR96 F. Forstnerič, J. Globevnik and J.-P. Rosay, Nonstraightenable complex lines in $\mathbf{C}^{2}$, Ark. Mat. 34 (1996), 97-101.

FR93 F. Forstnerič and J.-P. Rosay, Approximation of biholomorphic mappings by automorphisms of $\mathbb{C}^{n}$, Invent. Math. 112 (1993), 323-349.

GS77 V. Guillemin and S. Sternberg, Geometric asymptotics, Mathematical Surveys, vol. 14 (American Mathematical Society, Providence, RI, 1977).

Kod72 K. Kodaira, Holomorphic mappings of polydiscs into compact complex manifolds, J. Differential Geom. 6 (1971/72), 33-46.

Mat60 Y. Matsushima, Espaces homogènes de Stein des groupes de Lie complexes, Nagoya Math. J. 16 (1960), 205-218.

Mum74 D. Mumford, Hilberts 14th problem - The finite generation of subgroups such as rings of invariants, in Proc. conf. on Hilberts problems, DeKalb, 1974 (American Mathematical Society, Providence, RI, 1974).

Ros99 J.-P. Rosay, Automorphisms of $C^{n}$, a survey of Andersén-Lempert theory and applications, in Complex geometric analysis, Pohang, 1997, Contemporary Mathematics, vol. 222 (American Mathematical Society, Providence, RI 1999), 131-145.

RR88 J.-P. Rosay and W. Rudin, Holomorphic maps from $\mathbb{C}^{n}$ to $\mathbb{C}^{n}$, Trans. Amer. Math. Soc. 310 (1988), $47-86$.

TV00 Á. Tóth and D. Varolin, Holomorphic diffeomorphisms of semisimple Lie groups, Invent. Math. 139 (2000), 351-369.

Var99 D. Varolin, A general notion of shears, and applications, Michigan Math. J. 46 (1999), 533-553.

Var00 D. Varolin, The density property for complex manifolds and geometric structures. II, Internat. J. Math. 11 (2000), 837-847.

Var01 D. Varolin, The density property for complex manifolds and geometric structures, J. Geom. Anal. 11 (2001), 135-160.

Win01 J. Winkelmann, Large discrete sets in Stein manifolds, Math. Z. 236 (2001), 883-901.

Árpád Tóth toth@cs.elte.hu

Department of Analysis, Eötvös Loránd University, Budapest, Pázmány Péter 1/c, Hungary

Dror Varolin dror@math.sunysb.edu

Department of Mathematics, Stony Brook University, Stony Brook, NY 11794, USA 